\title{
Support Equalities Among Ribbon Schur Functions
}

\author{
Marisa Gaetz \\ Massachusetts Institute of Technology \\ Cambridge, MA, U.S.A. \\ mgaetz@mit.edu
}

\author{
Will Hardt \\ University of Wisconsin - Madison \\ Madison, WI, U.S.A. \\ whardt@wisc.edu
}

\author{
Shruthi Sridhar \\ Princeton University \\ Princeton, NJ, U.S.A. \\ ssridhar@math.princeton.edu
}

Submitted: Oct 8, 2018; Accepted: Aug 18, 2019; Published: Sep 13, 2019

(C) The authors. Released under the CC BY license (International 4.0).

\begin{abstract}
In 2007, McNamara proved that two skew shapes can have the same Schur support only if they have the same number of $k \times \ell$ rectangles as subdiagrams. This implies that two ribbons can have the same Schur support only if one is obtained by permuting row lengths of the other. We present substantial progress towards classifying when a permutation $\pi \in S_{m}$ of row lengths of a ribbon $\alpha$ produces a ribbon $\alpha_{\pi}$ with the same Schur support as $\alpha$; when this occurs for all $\pi \in S_{m}$, we say that $\alpha$ has full equivalence class. Our main results include a sufficient condition for a ribbon $\alpha$ to have full equivalence class. Additionally, we prove a separate necessary condition, which we conjecture to be sufficient.
\end{abstract}

Mathematics Subject Classifications: 05E05

\section{Introduction}

The question of when two skew diagrams yield equal skew Schur functions has been studied in detail. For instance, this question is addressed by Billera, Thomas, and van Willigenburg in [2], by McNamara and van Willigenburg in [8], and by Reiner, Shaw, and van Willigenburg in [10]. In this paper, we consider the related question of when two skew diagrams yield skew Schur functions such that when expanded, the same set of Schur functions appear with nonzero coefficient. In other words, we consider the question of when two skew diagrams have equal Schur support (Definition 1). This question has received less attention, with the most substantial progress coming from McNamara in 2007 [6] and from McNamara and van Willigenburg in 2011 [7]. 
Despite receiving relatively little attention, the study of Schur support equality and containment has potentially profound consequences. For instance, Schur support containment provides information regarding the Schur-positivity of differences of skew Schur functions. Specifically, defining the Schur-positivity order on skew shapes as $B \leqslant A$ if $s_{A}-s_{B}$ is Schur-positive, we have as a strong necessary condition for $B \leqslant A$ that the Schur support of $B$ is contained in that of $A$. The study of Schur-positive functions has applications to a broad range of mathematics, including to the representation theory of the symmetric group; McNamara and van Willigenburg have more on this in [8].

McNamara and van Willigenburg also explicitly determine the Schur support for a special class of skew shapes called equitable ribbons, which, by definition, are ribbons such that the lengths of any two nonempty rows differ by at most one and that the lengths of any two nonempty columns differ by at most one [7, Definition 3.4]. Furthermore, McNamara and van Willigenburg show that equitable ribbons are the maximal connected skew shapes in the Schur support containment order, and that there is a unique such maximal element with $\ell$ rows for each $\ell$ [7, Theorem 1.5].

Since the property of being equitable is preserved under permuting row lengths, it follows that equitable ribbons have the same Schur support under all permutations of row lengths, or, using our terminology, that equitable ribbons have full equivalence class (Definition 7). However, being equitable is not a necessary condition for a ribbon to have full equivalence class (see Theorem 25).

This definition of full equivalence class is motivated by the result of McNamara that any two skew diagrams with the same Schur support necessarily contain the same number of $k \times \ell$ rectangles as subdiagrams, for every $k, \ell \geqslant 1$ [6, Corollary 3.10]. From this, it follows easily that two ribbons can have equal Schur support only if one is obtained from the other by permuting its row lengths (Corollary 6). This reduces the problem of determining the support equivalence class of a ribbon to determining which permutations of the ribbon's row lengths preserve its support.

In this paper, we seek to address a portion of the Schur support equality problem by working to classify which ribbons have full equivalence class. In the next section, we provide preliminary information and address several edge cases to ensure the completeness and comprehensibility of our statements throughout the remainder of the paper. In Section 3, we prove our main result (Theorem 25), which states that a sufficient condition for a ribbon to have full equivalence class is that all 3 -subsets of its row lengths satisfy the strict triangle inequality (Definition 18); simply put, this condition requires that the largest of the three row lengths is strictly less than the sum of the other two. This is a weaker condition than being equitable; thus, this result generalizes the finding of McNamara and van Willigenburg in [7, Theorem 1.5] that equitable ribbons have full equivalence class. The key tools in our proof are the celebrated Littlewood-Richardson Rule [5] and an R-matrix algorithm originally due to Nakayashiki and Yamada [9] that is presented more clearly for our purposes by Inoue, Kuniba and Takagi in [4].

Section 4 gives a necessary condition on the row lengths for a ribbon to have full equivalence class (Theorem 27). This necessary condition is not simple to state, but in a forthcoming paper, Azenhas and Mamede [1] find additional meaning behind it, 


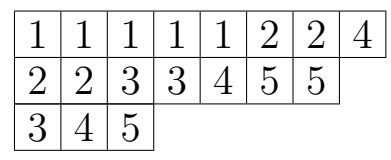

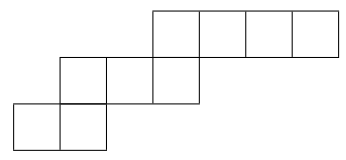

Figure 1: A semistandard Young tableau (left) and the ribbon $(4,3,2)$ (right).

showing that it coincides with their classification of when a monotone ribbon has full Schur support. (A skew shape $A$ with $n$ parts has full Schur support, by definition, when its Schur support is equal to its Schur interval - a subset of the partitions of $n$ defined with respect to the partitions $r(A)$ and $c(A)$ formed by the row lengths and column lengths of $A$, respectively [1].) We conjecture that our necessary condition for a ribbon to have full equivalence class is also sufficient, which we, along with Tran, have shown to be true for ribbons with 3 or 4 rows [3]. If our conjecture is true in general, then the work of Azenhas and Mamede would imply that a monotone ribbon has full Schur support if and only if it has full equivalence class. Finally, in Section 5, we give concluding remarks and pose problems for future consideration.

\section{Preliminaries}

We begin by establishing some basic definitions relating to Schur functions and ribbons. We will then introduce the Littlewood-Richardson rule, which is the underlying tool used in many of our proofs. Additionally, we will introduce an algorithm given by $R$-matrices, which will dictate a way to swap adjacent row lengths in a ribbon tableau, while preserving the content of the filling and semistandardness within the two swapped rows. This algorithm is an important part of our proof of a sufficient condition for full equivalence class (Theorem 25). Finally, we will consider several edge cases to allow for cleaner statements of results throughout the remainder of the paper.

\subsection{Schur Functions}

The Young diagram corresponding to a partition $\lambda=\left(\lambda_{1} \geqslant \lambda_{2} \geqslant \ldots \geqslant \lambda_{m}>0\right)$ of an integer $n$ is a collection of $n$ boxes arranged in left-aligned rows, where the $i^{\text {th }}$ row from the top has $\lambda_{i}$ boxes. A Young diagram filled with integers that increase weakly across rows and strictly down columns is called a semistandard Young tableau (SSYT) (Figure 1 (left)).

We use content to refer to the multiset of integers in the filling of a tableau. The content is denoted by a tuple $\nu=\left(\nu_{1}, \nu_{2}, \ldots, \nu_{k}\right)$, where $\nu_{i}$ is the number of $i$ 's in the filling of the tableau. For example, the content of the tableau from Figure 1 is $\nu=(5,4,3,3,3)$.

Schur functions are often considered to be the most important basis for the ring of symmetric functions. Schur functions are indexed by integer partitions, where the Schur function $s_{\lambda}$ corresponding to a partition $\lambda$ is defined as 


$$
s_{\lambda}\left(x_{1}, x_{2}, x_{3}, \ldots\right)=\sum_{\substack{T \\ \text { Shape } \lambda}} x^{T}=\sum_{T} \sum_{\substack{\text { SSYT } \\ \text { shape } \lambda}} x_{1}^{\nu_{1}} x_{2}^{\nu_{2}} x_{3}^{\nu_{3}} \ldots
$$

where $\nu_{i}$ is the number of occurrences of $i$ in $T$.

We can generalize this notion of Schur functions to apply to skew shapes, which are obtained by removing the Young diagram corresponding to the partition $\mu$ from the topleft corner of a larger Young diagram corresponding to the partition $\lambda$. Here, we require that the diagram for $\mu$ is contained in the diagram for $\lambda$, and we write the resulting skew shape as $\lambda / \mu$. When $\mu$ is the empty partition, $\lambda / \mu$ is said to be straight. Skew Schur functions have an analogous definition to that of straight Schur functions, where the sum in Equation 1 is instead over skew semistandard Young tableaux of shape $\lambda / \mu$.

Skew Schur functions have the nice property that they are Schur-positive, meaning that for any skew shape $\lambda / \mu$, we can write

$$
s_{\lambda / \mu}=\sum_{\nu} c_{\mu, \nu}^{\lambda} s_{\nu}
$$

where $\nu$ denotes a straight partition, and where all coefficients $c_{\mu, \nu}^{\lambda} \geqslant 0$. The coefficients $c_{\mu, \nu}^{\lambda}$ are called Littlewood-Richardson coefficients, and will play an important role in the Littlewood-Richardson rule (which we introduce in Theorem 9). Since such a decomposition of a skew Schur function into a linear combination of straight Schur functions is unique, the following notion is well-defined:

Definition 1. The Schur support of a skew shape $\lambda / \mu$, denoted $[\lambda / \mu]$, is defined as

$$
[\lambda / \mu]=\left\{\nu: c_{\mu, \nu}^{\lambda}>0\right\} .
$$

In other words, the support of a skew shape $\lambda / \mu$ is the set of straight shapes $\nu$ such that $s_{\nu}$ appears with nonzero coefficient in the expansion of $s_{\lambda / \mu}$ into a linear combination of straight Schur functions.

Remark 2. It is well known [11, Exercise 7.56(a)] that $\left[\alpha^{\circ}\right]=[\alpha]$, where $\alpha^{\circ}$ is the antipodal $\left(180^{\circ}\right)$ rotation of a ribbon $\alpha$.

\subsection{Ribbons}

A ribbon is a connected skew shape which does not contain a $2 \times 2$ rectangle as a subdiagram. Compositions of $n$ with $m$ parts are in natural bijection with ribbons with $n$ boxes and $m$ rows; hence, we will use the notation $\alpha=\left(\alpha_{1}, \alpha_{2}, \ldots, \alpha_{m}\right)$ to denote the ribbon with $m$ rows in which the length of the $i^{\text {th }}$ row is $\alpha_{i}$. For example, Figure 1 (right) shows the ribbon $(4,3,2)$.

Definition 3. Let $\alpha=\left(\alpha_{1}, \alpha_{2}, \ldots, \alpha_{m}\right)$ and $\alpha_{\pi}=\left(\alpha_{\pi^{-1}(1)}, \alpha_{\pi^{-1}(2)}, \ldots, \alpha_{\pi^{-1}(m)}\right)$ be ribbons, where $\pi \in S_{m}$. We say $\alpha_{\pi}$ is a permutation of $\alpha$.

Example 4. Figure 2 depicts all permutations of the ribbon $\alpha=(4,3,2)$. 


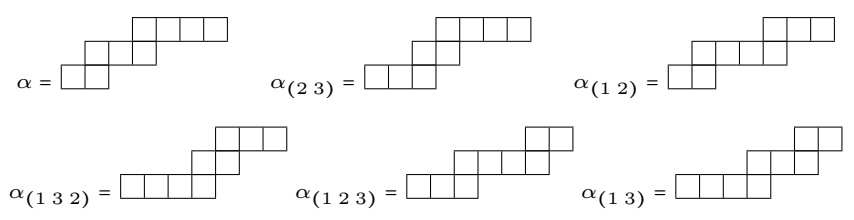

Figure 2: Permutations of the ribbon $\alpha=(4,3,2)$.

In [6], McNamara proves a useful necessary condition for Schur support equality:

Proposition 5 ([6, Corollary 3.10]). Let $A, B$ be skew shapes. If $[A]=[B]$, then $A$ and $B$ contain the same number of $k \times \ell$ rectangles as subdiagrams, for all $k, \ell \in \mathbb{N}$.

In particular, if $\alpha$ and $\beta$ are ribbons satisfying $[\alpha]=[\beta]$, Proposition 5 gives that $\alpha$ and $\beta$ contain the same number of $2 \times 1$ rectangles as subdiagrams, and hence have the same number of rows. Moreover, Proposition 5 gives that $\alpha$ and $\beta$ contain the same number of $1 \times k$ rectangles for every $k \in \mathbb{N}$. Consequently, $\alpha$ and $\beta$ have not only the same number of rows, but also the same multiset of row lengths. Thus, we have the following:

Corollary 6. Let $\alpha, \beta$ be ribbons. If $[\alpha]=[\beta]$, then $\beta$ is a permutation of $\alpha$.

Definition 7. A ribbon $\alpha=\left(\alpha_{1}, \alpha_{2}, \ldots, \alpha_{m}\right)$ has full equivalence class if $[\alpha]=\left[\alpha_{\pi}\right]$ for all permutations $\pi \in S_{m}$.

For instance, the ribbon $\alpha=(4,3,2)$ from Example 4 has full equivalence class, since all of its permutations have support $\{(7,2),(7,1,1),(6,3),(6,2,1),(5,4),(5,3,1),(5,2,2)$, $(4,4,1),(4,3,2)\}$.

\subsection{Yamanouchi Words and Tableaux}

We now introduce the concepts of Yamanouchi words and Yamanouchi tableaux, which will be essential for using and defining our main tool for proving support equality - the Littlewood-Richardson rule.

A Yamanouchi word is a word with the property that all of its prefixes contain no more $(i+1)$ 's than $i$ 's, for all integers $i \geqslant 1$. For our purposes, we are concerned with the reverse reading word (henceforth $R R W$ ) of a tableau, which reads right-to-left across rows and top-to-bottom from one row to the next. A Yamanouchi tableau is a tableau whose RRW is a Yamanouchi word.

Example 8. The tableau depicted in Figure 3 is Yamanouchi because each prefix of its RRW (i.e. of 112213321) contains no more 2's than 1's, and contains no more 3's than 2's.

\subsection{Littlewood-Richardson Tableaux}

Littlewood-Richardson tableaux (which we often abbreviate as LR-tableaux) are tableaux which are both semistandard and Yamanouchi. These tableaux play an important role in the Littlewood-Richardson rule, which we are now ready to state. 


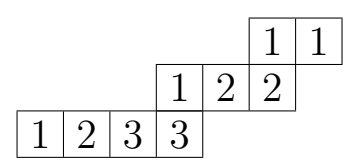

Figure 3: Young tableau with RRW 112213321.

Theorem 9 (Littlewood-Richardson rule [5] or [11, Theorem A.1.3.3]). If

$$
s_{\lambda / \mu}=\sum_{\nu} c_{\mu, \nu}^{\lambda} s_{\nu}
$$

then the number of Littlewood-Richardson tableaux of shape $\lambda / \mu$ and content $\nu$ is $c_{\mu, \nu}^{\lambda}$.

The following corollary follows immediately from Theorem 9 and the definition of Schur support (Definition 1), and will be more directly applicable to the proofs in the remainder of the paper.

Corollary 10. Let $\nu$ be a straight shape and $\lambda / \mu$ a skew shape. Then $\nu \in[\lambda / \mu]$ if and only if there exists a Littlewood-Richardson tableau of shape $\lambda / \mu$ and content $\nu$.

Example 11. The tableau in Figure 3 is both semistandard and Yamanouchi, with content $(4,3,2)$. It follows from Corollary 10 that the straight shape $(4,3,2)$ is in the support of the ribbon with row lengths $(2,3,4)$.

\section{$2.5 \quad$ R-Matrices}

We will now introduce an algorithm which will be instrumental in proving our sufficient condition for a ribbon to have full equivalence class. The $R$-matrix algorithm, described by Inoue, Kuniba and Takagi in [4, Section 2.2.3], provides a way to swap two consecutive row lengths in an arbitrary ribbon with a semistandard filling so that the filling within the two rows remains semistandard and has the same content as before. Note, however, that semistandardness of the ribbon as a whole is not necessarily preserved.

Let $A$ be a ribbon tableau of shape $\alpha=\left(\alpha_{1}, \ldots, \alpha_{m}\right)$, and assume that $\alpha_{j}>\alpha_{j+1}$ (note that if $\alpha_{j}<\alpha_{j+1}$, we can swap $\alpha_{j}$ and $\alpha_{j+1}$ by performing this algorithm on the antipodal rotation $\alpha^{\circ}$, and then taking the antipodal rotation of the result). The algorithm proceeds as follows.

1. Convert the fillings of rows $j$ and $j+1$ to a "box-ball system" as follows. Let $e$ denote the maximal entry in the $j^{t h}$ and $(j+1)^{s t}$ rows. Create two length-e columns of boxes. If $\left(\nu_{1}, \ldots, \nu_{e}\right)$ is the content of the $j^{\text {th }}$ row, place $\nu_{i}$ balls in the $i^{\text {th }}$ box from the top of the left column for each $1 \leqslant i \leqslant e$. Do the same for the $(j+1)^{s t}$ row in the right column.

2. For each ball on the right (in any order), connect it to an unconnected ball on the left strictly above it which is as low as possible. If there is no such ball on the left, connect it to the lowest unconnected ball on the left. 
3. Shift all unconnected balls on the left horizontally to the right.

4. Convert this box-ball system back into rows of a ribbon tableau by doing the inverse of the operation described in (1).

Remark 12. The result of the $R$-matrix algorithm depends only on which of the balls on the left become connected to a ball on the right. As a result, when executing the $R$-matrix algorithm, the order in which the balls on the right are connected to balls on the left does not matter.

Proposition 13. The $R$-matrix algorithm applied to a two-row semistandard ribbon tableau A outputs a two-row semistandard ribbon tableau.

Proof. Since $A$ is semistandard, there is at least one entry $a$ in the second row of $A$ that is strictly greater than the smallest entry in the first row of $A$. After $A$ has been converted into a box-ball system, this means that there is at least one ball on left strictly above the ball corresponding to $a$ on the right. In light of Remark 12, we can then assume without loss of generality that the ball corresponding to $a$ on the right is connected to a ball above it on the left (as opposed to being connected to the lowest ball ball overall on the left). Recall that this ball above $a$ on the left corresponds to an entry less than $a$ from the top row. Since only the unconnected balls are slid over to the right column, this means that at the completion of the algorithm, the top row still has an entry that is strictly less than $a$.

The rows in the outputted tableau are weakly increasing simply because of how we convert a box-ball system back into a tableau. Then the above argument establishes that the $2 \times 1$ rectangle where the rows overlap in the outputted tableau is semistandard, so the proof is complete.

Although the $R$-matrix algorithm preserves semistandardness when applied to tworow ribbon tableaux, we would often like to apply the algorithm to two-row subtableaux of semistandard ribbon tableaux. Suppose the $R$-matrix algorithm is applied to rows $i$ and $i+1$ of a ribbon tableau $A$, and call the resulting tableau $A_{(i+1)}$. Then the two-row subtableau formed by rows $i$ and $i+1$ of $A_{(i+1)}$ will be semistandard (Proposition 13), but $A_{(i i+1)}$ will not necessarily be semistandard (Example 15).

Example 14. [4, Sect. 2.2.3] We borrow an example from Inoue, Kuniba and Takagi. Suppose we have a ribbon whose $j^{t h}$ and $(j+1)^{s t}$ rows are as in Figure 4 (left). Steps 1-3 of the $R$-matrix algorithm as applied to this partial tableau are depicted in Figure 4 (middle). Notice that the only ball movement is two balls in the third box from the top shifting from the left to the right, as these were the only two unconnected balls on the left. After applying step 4 of the $R$-matrix algorithm, we obtain the partial tableau depicted in Figure 4 (right). Notice that the row lengths have swapped, while the content and semistandardness of the filling has been preserved, as promised. Moreover, in this example, both the rightmost entry in the $j^{\text {th }}$ row and the leftmost entry in the $(j+1)^{s t}$ row remain unchanged, meaning semistandardness of the entire ribbon would be preserved. This will not always be the case, as will be illustrated in the next example. 

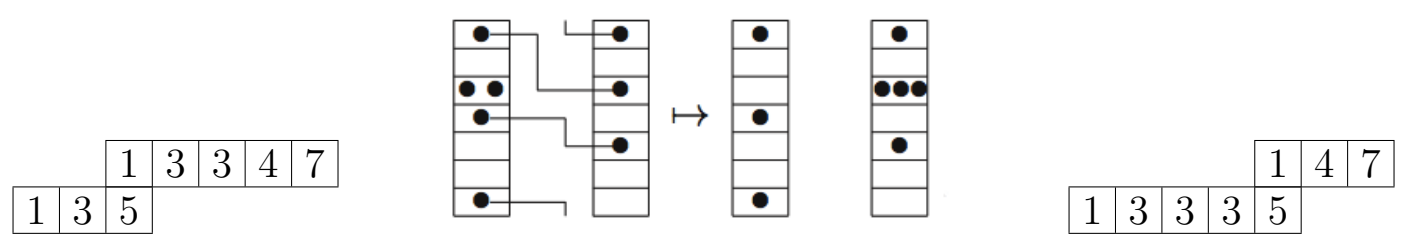

Figure 4: R-matrix algorithm in action
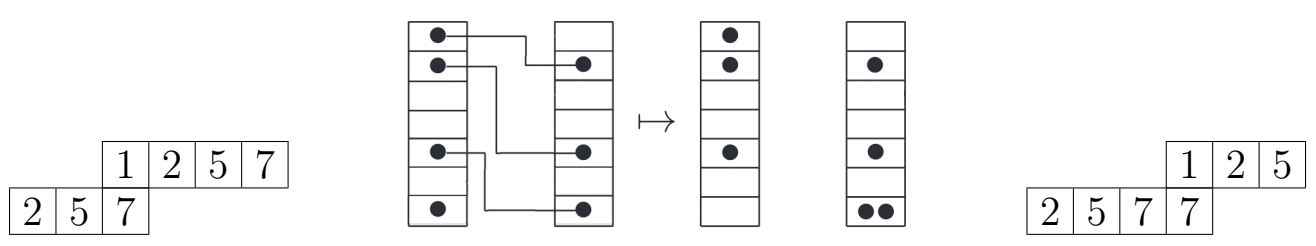

Figure 5: R-matrix algorithm that changes top right entry

Example 15. Consider a ribbon whose $j^{\text {th }}$ and $(j+1)^{\text {st }}$ rows are as in Figure 5 (left). An application of steps 1-3 of the $R$-matrix algorithm to these rows is shown in Figure 5 (middle), with the resulting partial tableau shown in Figure 5 (right). As in the previous example, both the content of and semistandardness between the $j^{\text {th }}$ and $(j+1)^{\text {st }}$ rows have been preserved. However, the rightmost entry of the $j^{\text {th }}$ row is changed from a 7 to a 5. As a result, we see that if the leftmost entry in the $(j-1)^{\text {st }}$ row is, say, a 6 , then semistandardness between the $(j-1)^{s t}$ and $j^{\text {th }}$ rows would not be preserved by this application of the $R$-matrix algorithm.

\subsection{Edge Cases}

To conclude the Preliminaries, we classify which ribbons have full equivalence class in a couple of edge cases: ribbons with fewer than three rows and ribbons with row(s) of length one. Addressing these edge cases here will allow for cleaner statements of our main results later.

Proposition 16. Any ribbon $\alpha$ with at most two rows has full equivalence class.

Proof. If $\alpha$ has one row, then it trivially has full equivalence class. If $\alpha$ has two rows, then $\alpha$ has full equivalence class by Remark 2 .

Proposition 17. Let $\alpha=\left(\alpha_{1}, \alpha_{2}, \ldots, \alpha_{m}\right)$ be a ribbon with $m \geqslant 3$. If $\alpha_{i}=1$ for some $i \epsilon$ $\{1,2, \ldots, m\}$, then $\alpha$ has full equivalence class if and only if $\alpha_{j}=1$ for all $j \in\{1,2, \ldots, m\}$.

Proof. $(\Leftarrow)$ : Trivially, if every row has length one, then $\alpha$ has full equivalence class.

$(\Rightarrow)$ : Arguing by contrapositive, we first consider the case where $\alpha$ has exactly $m-1$ rows of length one (so only one "long" row of length $>1$ ). Let $\alpha_{\beta}$ be a permutation of $\alpha$ in which the long row is the first row and $\alpha_{\gamma}$ a permutation of $\alpha$ in which the long row is the second row. Then we notice that $\alpha_{\beta}$ has an $m \times 1$ rectangle ranging vertically over all rows; however, $\alpha_{\beta}$ does not contain an $m \times 1$ rectangle. So by Proposition $5, \alpha$ does not have full equivalence class. 
Now assume that $\alpha$ has exactly $k$ rows which are of length 1 , where $1 \leqslant k \leqslant m-2$. Let $\alpha_{\sigma}$ be any permutation of $\alpha$ such that the first $k$ rows of $\alpha_{\sigma}$ have length 1 . Let $\alpha_{\pi}$ be the permutation of $\alpha$ obtained from $\alpha_{\sigma}$ by swapping the lengths of the first and $(k+1)^{s t}$ rows. Notice that $\alpha_{\pi}$ has a $(k+2) \times 1$ rectangle as a subdiagram, whereas $a_{\sigma}$ does not. Hence, by Proposition 5, $\alpha$ does not have full equivalence class.

\section{A Sufficient Condition}

In this section, we prove a sufficient condition for a ribbon to have full equivalence class; the statement of this condition requires the following notion:

Definition 18. A set $\{x, y, z\}$ of three integers $x \leqslant y \leqslant z$ satisfies the strict triangle inequality if $z<x+y$.

Theorem 25. Let $\alpha=\left(\alpha_{1}, \alpha_{2}, \ldots, \alpha_{m}\right)$ be a ribbon with each $\alpha_{i} \geqslant 2$ and $m \geqslant 3$. If all 3-subsets of $\left\{\alpha_{i}\right\}_{i=1}^{m}$ satisfy the strict triangle inequality, then $\alpha$ has full equivalence class.

Towards the goal of proving this sufficient condition for a ribbon to have full equivalence class, we begin with two lemmas. In Lemma 19, we prove that the $R$-matrix algorithm preserves the Yamanouchi property of ribbon LR-tableaux. Lemma 20 shows that the $R$-matrix algorithm, when applied to a ribbon LR-tableau, preserves some of the semistandardness of the tableau. In particular, if we use the $R$-matrix algorithm to swap rows $j$ and $j+1$ of a ribbon LR-tableau $A$, then semistandardness is preserved between the $(j+1)^{s t}$ and $(j+2)^{n d}$ rows of $A$.

We use the results of these two lemmas to show in Theorem 21 that given three adjacent rows satisfying the strict triangle inequality (with the second row longer than the third), the bottom two of the three adjacent row lengths can be swapped while preserving the Yamanouchi property and semistandardness of the tableau. Extending this condition to all row length triples, we obtain a sufficient condition for a ribbon to have full equivalence class (Theorem 25).

Lemma 19. Let $A$ be a ribbon LR-tableau of shape $\alpha=\left(\alpha_{1}, \ldots, \alpha_{m}\right)$ and let $i \in\{1,2, \ldots$, $m-1\}$. If $A_{(i+1)}$ is the ribbon tableau of shape $\alpha_{(i i+1)}$ that results from applying the $R$-matrix operation to rows $i$ and $i+1$ of $A$, then $A_{(i+1)}$ is a Yamanouchi tableau.

Proof. Since $A$ is Yamanouchi, we only need to show that the prefixes of the RRW up through the $i^{\text {th }}$ and $(i+1)^{s t}$ rows of $A_{(i+1)}$ are Yamanouchi. Consider the $i^{\text {th }}$ and $(i+$ $1)^{s t}$ rows of $A$. Fix any positive integer $j$ and assume that $j$ and $j+1$ appear in the aforementioned rows as follows (with $a, b, c, d$ possibly zero):

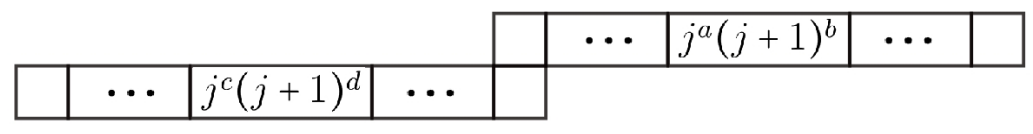


Let $\eta_{j}$ and $\eta_{j+1}$ denote the number of $j$ 's and $(j+1)$ 's, respectively, in the RRW of $A_{(i i+1)}$ by the end of the $(i-1)^{\text {st }}$ row. Since $A$ is assumed to be Yamanouchi, we have that $\eta_{j}-\eta_{j+1} \geqslant b$ and $\eta_{j}-\eta_{j+1} \geqslant b+d-a$.

We now execute the R-matrix algorithm. Let $x$ be the number of balls in the $j^{\text {th }}$ box on the left that are connected to a ball on the right. Similarly,let $y$ be the number of balls in the $(j+1)^{s t}$ box on the left that are connected to a ball on the right. Notice that $x \geqslant \min (a, d)$. Following the $R$-matrix algorithm, $j$ and $j+1$ occur in the $i^{\text {th }}$ and $(i+1)^{s t}$ rows of $A_{(i i+1)}$ as follows:

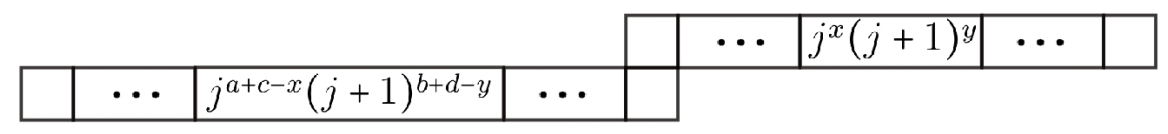

Let $R$ be the word $(j+1)^{y} j^{x}(j+1)^{b+d-y} j^{a+c-x}$. Define the function $r(n)$ to be the number of $(j+1)$ 's minus the number of $j$ 's in the first $n$ elements of the word $R$. We have left to show that $r$ never exceeds $\eta_{j}-\eta_{j+1}$. Since $r$ is maximal at $y$ and at $b+d+x$, it suffices to show that $r(y) \leqslant \eta_{j}-\eta_{j+1}$ and $r(b+d+x) \leqslant \eta_{j}-\eta_{j+1}$.

Notice that $r(y)=y$ and $r(b+d+x)=(y+(b+d-y))-x=b+d-x$. Since $y \leqslant b \leqslant \eta_{j}-\eta_{j+1}$, we have that $r(y) \leqslant \eta_{j}-\eta_{j+1}$. For $r(b+d+x)$, we consider two cases. If $x \geqslant d$, then $r(b+d+x)=b+d-x \leqslant b \leqslant \eta_{j}-\eta_{j+1}$, as desired. On the other hand, if $x<d$, then since $x \geqslant \min (a, d)$ (as noted above), we have $x \geqslant a$. Then $r(b+d+x)=b+d-x \leqslant b+d-a \leqslant \eta_{j}-\eta_{j+1}$. This completes the proof.

We have just shown that the $R$-matrix algorithm preserves the Yamanouchi property in ribbon LR-tableaux. Recall from the Preliminaries that the $R$-matrix operation, as applied to a two-row subtableau of a ribbon LR-tableau, preserves semistandardness within the subtableau; however, semistandardness of the entire ribbon is not necessarily preserved. Our next lemma shows that applying the $R$-matrix algorithm to rows $i$ and $i+1$ of a ribbon LR-tableau cannot increase the leftmost element in the $(i+1)^{s t}$ row. This result is a step towards establishing how we might use the $R$-matrix algorithm to swap row lengths, while preserving the semistandardness of the entire ribbon.

Lemma 20. Let $A$ be a ribbon LR-tableau of shape $\alpha=\left(\alpha_{1}, \ldots, \alpha_{m}\right)$ and let $i \in\{1,2, \ldots$, $m-1\}$. Denote by $A_{(i i+1)}$ the ribbon of shape $\alpha_{(i i+1)}$ obtained by applying the $R$-matrix algorithm to rows $i$ and $i+1$ of $A$. Let $x$ be the leftmost element of the $(i+1)^{\text {st }}$ row of $A$ and let $y$ be the leftmost element of the $(i+1)^{\text {st }}$ row of $A_{(i i+1)}$. Then $y \leqslant x$.

Proof. Since $x$ is in the $(i+1)^{s t}$ row of $A$, by the $R$-matrix algorithm, there is also an $x$ in the $(i+1)^{s t}$ row of $A_{(i i+1)}$. The result follows because the $(i+1)^{s t}$ row of $A_{(i i+1)}$ is weakly increasing.

The remaining way in which $A_{(i i+1)}$ may fail to be semistandard is for the number in the rightmost box of the $i^{\text {th }}$ row of $A_{(i i+1)}$ to be less than or equal to the number in the leftmost box of the $(i-1)^{s t}$ row. This last obstacle is the main focus of the following proof. 


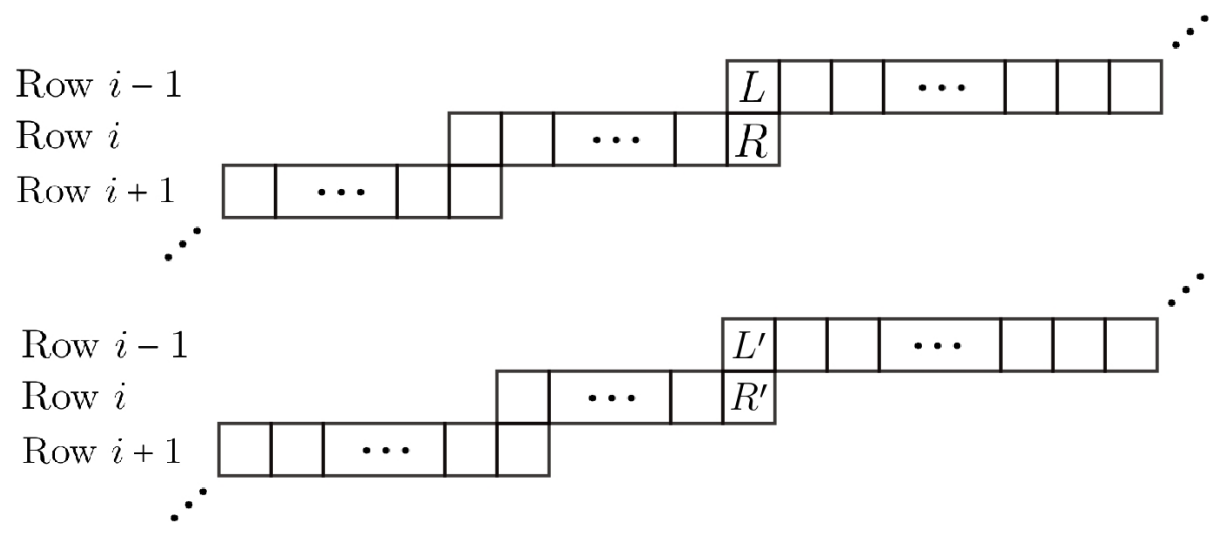

Figure 6: Box labellings of $A$ (top) and $A_{(i i+1)}$ (bottom).

Theorem 21. Let $\alpha=\left(\alpha_{1}, \alpha_{2}, \ldots, \alpha_{m}\right)$ be a ribbon, where each $\alpha_{i} \geqslant 2$ and $m \geqslant 3$. Suppose that $\alpha_{i+1}<\alpha_{i}<\alpha_{i-1}+\alpha_{i+1}$ for some $1 \leqslant i \leqslant(m-1)$, where $\alpha_{0}=\infty$ for notational convenience. Then $[\alpha] \subseteq\left[\alpha_{(i i+1)}\right]$.

Proof. Let $A$ be a ribbon LR-tableau with shape $\alpha$ and content $\nu$. By Corollary 10, it suffices to show that there is some ribbon LR-tableau with shape $\alpha_{(i i+1)}$ and content $\nu$.

Applying the $R$-matrix algorithm to rows $i$ and $i+1$ of $A$ yields a ribbon tableau $A_{(i i+1)}$ of shape $\alpha_{(i i+1)}$ and content $\nu$. By Lemma 19, $A_{(i i+1)}$ is Yamanouchi. If $A_{(i i+1)}$ is also semistandard, we are done. Recall from Proposition 13 that applying the $R$-matrix algorithm to rows $i$ and $i+1$ of $A$ preserves the semistandardness within the two rows. Moreover, we have by Lemma 20 that the leftmost entry in the $(i+1)^{\text {st }}$ row of $A_{(i i+1)}$ is not greater than that of $A$. Consequently, if $i=1$, then $A_{(i i+1)}$ is semistandard, and we are done. Supposing $i>1$, the only way in which $A_{(i i+1)}$ can fail to be semistandard is if the rightmost entry in the $i^{\text {th }}$ row of $A_{(i i+1)}$ is less than or equal to the leftmost entry in the $(i-1)^{s t}$ row of $A_{(i i+1)}$. Assume that this is the case (i.e. that $A_{(i i+1)}$ is not semistandard). In the remainder of the proof, we show that $A_{(i i+1)}$ can be modified to produce a tableau which is both semistandard and Yamanouchi. Each modification will preserve both shape and content, so the resulting tableau will still have shape $\alpha_{(i i+1)}$ and content $\nu$.

Let $L$ (resp. $L^{\prime}$ ) denote the leftmost box in the $(i-1)^{s t}$ row of $A$ (resp. $\left.A_{(i i+1)}\right)$. Similarly, let $R$ (resp. $R^{\prime}$ ) denote the rightmost box in the $i^{\text {th }}$ row of $A$ (resp. $\left.A_{(i+1)}\right)$ ). These labellings are shown in Figure 6. Let $\ell, r, \ell^{\prime}$, and $r^{\prime}$ be the entries in boxes $L, R$, $L^{\prime}$, and $R^{\prime}$, respectively. In the following claim, we argue that we can assume the content of $A_{(i i+1)}$ to be of a specific form, showing that in all other cases, $A_{(i i+1)}$ can be modified to produce a ribbon LR-tableau.

Claim 22. We can assume that every entry in the $i^{\text {th }}$ and $(i-1)^{\text {st }}$ rows (except possibly the rightmost in the $(i-1)^{\text {st }}$ row) of $A_{(i+1)}$ is an $r^{\prime}$ (see Figure 8).

Proof of Claim 22. By our assumption that $A_{(i i+1)}$ is not semistandard, we have that $\ell^{\prime} \geqslant r^{\prime}$. If $\ell^{\prime}>r^{\prime}$, we can simply swap the positions of $\ell^{\prime}$ and $r^{\prime}$ to obtain a semistandard 


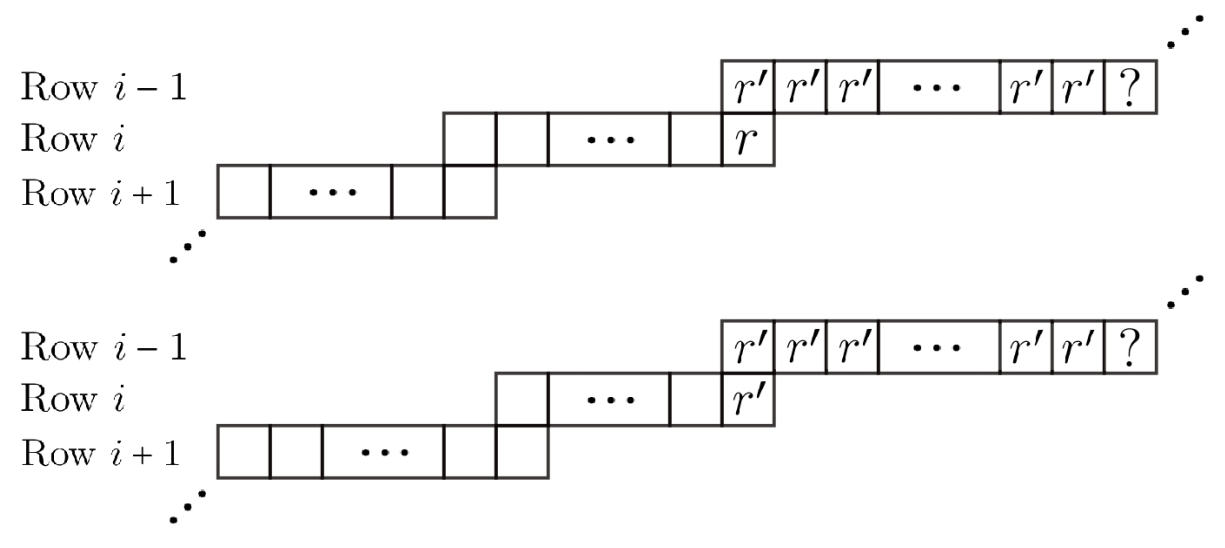

Figure 7: Assumed entries of $A$ (top) and $A_{(i i+1)}$ (bottom).

tableau. This swap preserves the Yamanouchi property, as the RRW of the resulting tableau can be obtained from that of $A_{(i i+1)}$ without moving $i$ to before $i-1$ for any $2 \leqslant i \leqslant m$. We can therefore assume that $\ell^{\prime}=r^{\prime}$.

Let $w$ denote the leftmost entry in the $(i-1)^{s t}$ row of $A_{(i i+1)}$ that is greater than $r^{\prime}=\ell^{\prime}$ (if such a $w$ exists). If $w$ is not the rightmost entry of the row, then we can swap it with the $r^{\prime}$ in box $R^{\prime}$, yielding a semistandard tableau $A_{(i i+1), w}$. That $A_{(i i+1), w}$ is Yamanouchi follows easily from the fact that $A_{(i i+1)}$ is Yamanouchi.

Therefore, in the case that $w$ exists and is not the rightmost entry of row $i-1$, we have obtained an LR-tableau with content $\nu$. Thus we may assume that all entries, except possibly the rightmost, of the $(i-1)^{s t}$ row of $A_{(i i+1)}$ equal $r^{\prime}=\ell^{\prime}$. Recall that $A$ and $A_{(i i+1)}$ differ only in their $i^{\text {th }}$ and $(i+1)^{s t}$ rows. Therefore, our assumptions regarding the entries of the $(i-1)^{s t}$ row of $A_{(i i+1)}$ apply also to the $(i-1)^{s t}$ row of $A$. These assumptions are depicted in Figure 7.

Let $x$ be the rightmost entry of the $i^{\text {th }}$ row of $A_{(i i+1)}$ that is less than $r^{\prime}$ (if such an $x$ exists). Unless $x$ is the leftmost entry of the row, we can swap $x$ with the $r^{\prime}$ in box $L^{\prime}$ to obtain a semistandard tableau. As before, the resulting tableau is Yamanouchi. We can therefore assume that all entries in the $i^{\text {th }}$ row of $A_{(i i+1)}$, except possibly the leftmost, are equal to $r^{\prime}$. We proceed to show that in fact, we may also assume that the leftmost entry of this row is $r^{\prime}$.

Since $A$ is semistandard, the element $r$ in box $R$ of $A$ must be strictly greater than $r^{\prime}$. Recall that the $i^{\text {th }}$ and $(i+1)^{s t}$ rows of $A$ and $A_{(i i+1)}$ have the same content. Noting that all entries in the $i^{t h}$ row of $A_{(i i+1)}$ are at most $r^{\prime}$, there must therefore be an $r$ in the $(i+1)^{s t}$ row of $A_{(i i+1)}$.

Now, let $y$ denote the leftmost entry of the $i^{\text {th }}$ row of $A_{(i i+1)}$, and consider the case where $y \neq r^{\prime}$. Using that the $R$-matrix algorithm preserves semistandardness within the rows it swaps (Proposition 13), we have that $y<r^{\prime}$. We can therefore swap this $y$ with the $r^{\prime}$ in box $L^{\prime}$ of $A_{(i i+1)}$ to obtain a semistandard tableau. As before, this swap does not violate the Yamanouchi property. We may therefore assume that $y=r^{\prime}$. By the above arguments, we may now assume that the entries of $A_{(i i+1)}$ are as shown in Figure 


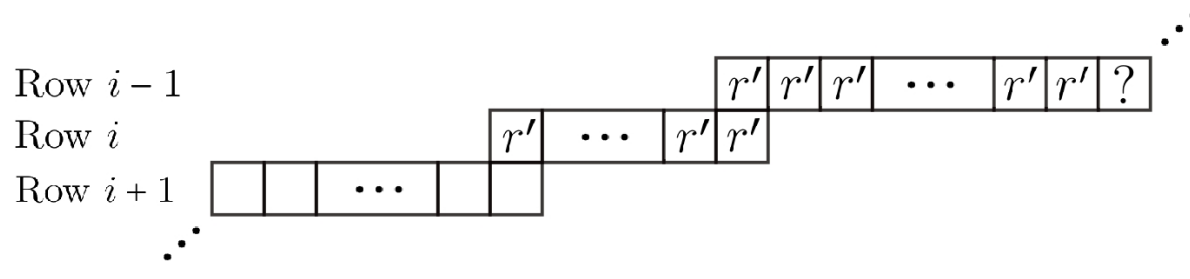

Figure 8: Assumed entries of $A_{(i i+1)}$.

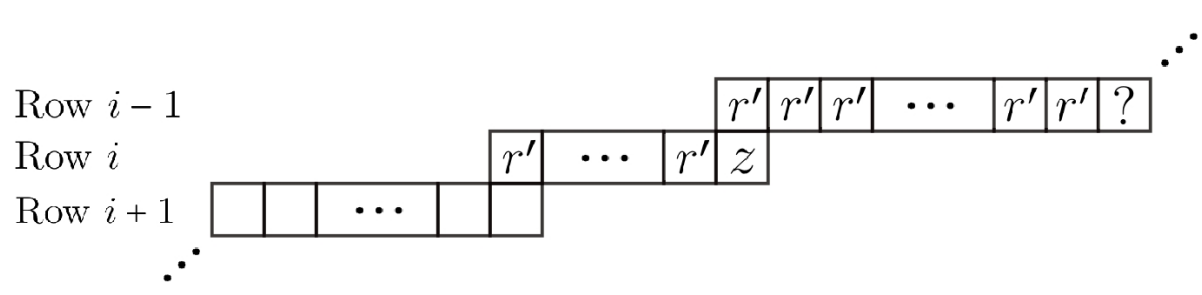

Figure 9: Entries of $A_{(i+1)}^{z}$.

8, completing the proof of Claim 22 .

We continue with the proof of Theorem 21. In this next portion of the proof, the idea is to swap the rightmost $r^{\prime}$ in the $i^{\text {th }}$ row of $A_{(i i+1)}$ with the appropriate entry from the row below to obtain an LR-tableau. In doing so, our assumptions regarding the content of $A_{(i+1)}$ become even stronger. In particular, consider the leftmost box in the $(i+1)^{s t}$ row of $A_{(i i+1)}$ with entry greater than $r^{\prime}$, and call this entry $z$.

Claim 23. We can assume that every entry in the $(i+1)^{\text {st }}$ row of $A_{(i i+1)}$ is a $z$ (see Figure 11).

Proof of Claim 23. We first argue that the leftmost box in the $(i+1)^{\text {st }}$ row of $A_{(i i+1)}$ with entry greater than $r^{\prime}$ cannot be the rightmost box of the $(i+1)^{s t}$ row of $A_{(i i+1)}$. Suppose, for the sake of contradiction, that the former box is in fact the rightmost box of the $(i+1)^{s t}$ row of $A_{(i i+1)}$. Then the $i^{t h}$ and $(i+1)^{s t}$ rows of $A$ and $A_{(i i+1)}$ have only one entry that is greater than $r^{\prime}$ (namely, $z$ ). In particular, using our previous notation (see Figure 7), we have that $r=z$. Since there is only one element greater than $r^{\prime}$ in these two rows, we have by the semistandardness of $A$ that the leftmost element in the $i^{\text {th }}$ row of $A$ is strictly less than $r^{\prime}$ (otherwise $r^{\prime}$ would occur twice in the same column of $A$ ). However, the $R$-matrix algorithm makes it impossible for both $z$ and the entry less than $r^{\prime}$ to be moved to the $(i+1)^{s t}$ row, while $r^{\prime}$ s remain in the $i^{\text {th }}$ row. We therefore conclude that the leftmost box in the $(i+1)^{s t}$ row of $A_{(i i+1)}$ with entry greater than $r^{\prime}$ is not the rightmost box of the row. Consequently, swapping the leftmost $z$ of the $(i+1)^{s t}$ row of $A_{(i i+1)}$ with the $r^{\prime}$ in box $R^{\prime}$ of $A_{(i i+1)}$ will produce a semistandard tableau $A_{(i i+1)}^{z}$ (see Figure 9). If $A_{(i+1)}^{z}$ is Yamanouchi, then the proof is complete. We now show that $A_{(i+1)}^{z}$ can only fail to be Yamanouchi in a very narrow set of cases. 


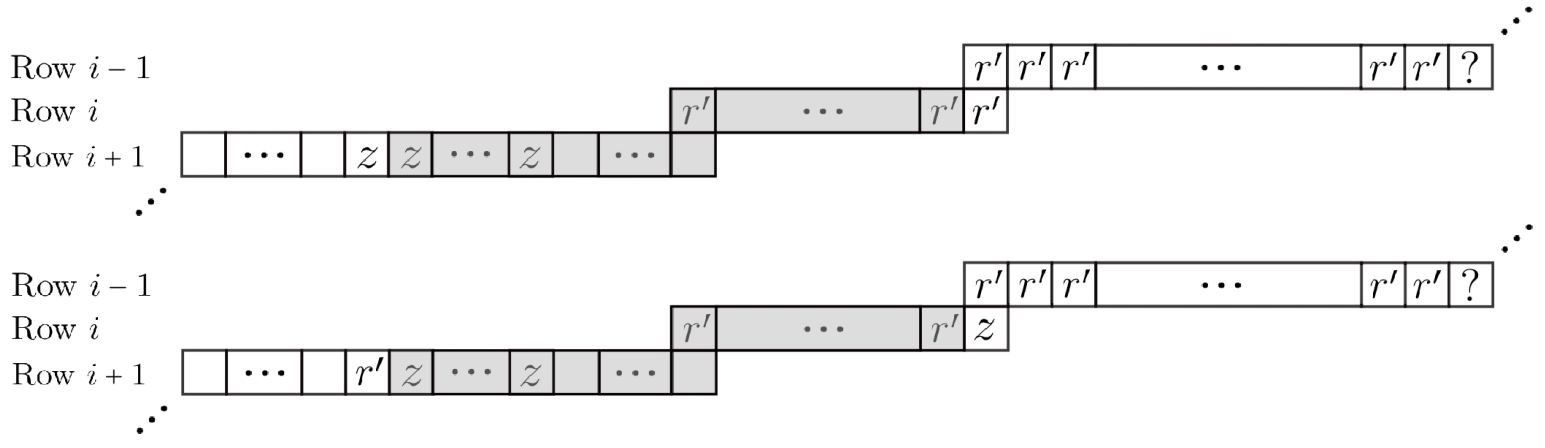

Figure 10: This figure (top) depicts entries of $A_{(i+1)}$ as well as subwords of the RRW of $A_{(i i+1)}$. The gray shading indicates entries corresponding to the subword $X$, while the "?" indicates the last element of the subword $W$. This figure (bottom) depicts the analogous entries and subwords of $A_{(i i+1)}^{z}$.

Let $X$ denote the subword of the RRW of $A_{(i i+1)}$ and $A_{(i i+1)}^{z}$ formed by the entries strictly between the entries we swapped to obtain $A_{(i+1)}^{z}$ from $A_{(i i+1)}$ (see Figure 10). If $z \neq r^{\prime}+1$, then this subword does not contain either $r^{\prime}+1$ or $z-1$. Consequently, this swap preserves the Yamanouchi property when $z \neq r^{\prime}+1$. We may therefore assume that $z=r^{\prime}+1$. Thus, given that $A_{(i i+1)}$ is Yamanouchi, the only way in which $A_{(i i+1)}^{z}$ can fail to be Yamanouchi is if the following phenomenon occurs: within the subword $X$, the number of $z$ 's in the RRW of $A_{(i i+1)}^{z}$ overtakes the number of $r^{\prime}$ 's in the RRW of $A_{(i i+1)}^{z}$. To understand the set of cases in which this phenomenon can occur, let us further investigate the RRW of $A_{(i i+1)}^{z}$.

Noting that the subword $X$ begins with $\alpha_{i+1}-1$ occurrences of $r^{\prime}$, it suffices to investigate the portion of $X$ determined by elements in the $(i+1)^{s t}$ row of $A_{(i i+1)}^{z}$. If there are no $z$ 's in this row, then there is nothing to check. Therefore, assume that there is at least one $z$ in the $(i+1)^{s t}$ row of $A_{(i+1)}^{z}$. By the semistandardness of $A_{(i i+1)}^{z}$, this means that there is exactly one contiguous string of $z$ 's in this portion of $X$ (say, of length $k$ ). Moreover, by definition of $X$, this contiguous string is a suffix of $X$.

Let $Z$ denote the prefix of the RRW of $A_{(i i+1)}^{z}$ formed by truncating after $X$. It follows that if $A_{(i i+1)}^{z}$ violates the Yamanouchi property, then so does $Z$. Also, let $W$ denote the prefix of the RRW of $A_{(i i+1)}$ and $A_{(i i+1)}^{z}$ of length $\sum_{j=1}^{i-2}\left(\alpha_{j}\right)+1$ (i.e. the prefix ending immediately after the rightmost element of the $(i-1)^{s t}$ row). Note that we can write $Z=W\left(r^{\prime}\right)^{\alpha_{i-1}-1} z X$ (see Figure 10). The prefix of $A_{(i i+1)}$ of the same length can be written as $W\left(r^{\prime}\right)^{\alpha_{i-1}} X$ (see Figure 10).

Let $d$ be the number of $r^{\prime}$ 's that occur in $W$ minus the number of $z^{\prime}$ 's that occur in $W$. Since $W$ is Yamanouchi and $z=r^{\prime}+1$, we have that $d \geqslant 0$. Recalling that $A_{(i i+1)}$ is Yamanouchi, we have

$$
d+\left(\alpha_{i-1}-1\right)+\alpha_{i+1}-(k+1) \geqslant 0 .
$$


Row $i-2$

Row $i-1$

Row $i$

Row $i+1$

\begin{tabular}{|l|l|l|l|l|}
\hline$r^{\prime}$ & $\cdots$ & $r^{\prime}$ & $r^{\prime}$ \\
\hline
\end{tabular}

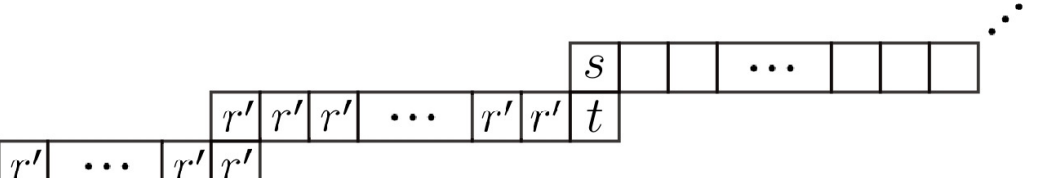

Figure 11: Assumed entries of $A_{(i+1)}$.

Suppose that $A_{(i+1)}^{z}$ is not Yamanouchi. Then

$$
d+\left(\alpha_{i-1}-1\right)-1+\left(\alpha_{i+1}-1\right)-k=\left(d+\left(\alpha_{i-1}-1\right)+\alpha_{i+1}-(k+1)\right)-1<0 .
$$

Together, (2) and (3) give that $d+\left(\alpha_{i-1}-1\right)+\alpha_{i+1}-(k+1)=0$. Noting that $k+1 \leqslant \alpha_{i} \leqslant$ $\alpha_{i-1}+\alpha_{i+1}-1$, we have

$$
\begin{aligned}
d & =d+\left(\alpha_{i-1}+\alpha_{i+1}-1\right)-\left(\alpha_{i-1}+\alpha_{i+1}-1\right) \\
& \leqslant d+\alpha_{i-1}+\alpha_{i+1}-1-\alpha_{i} \\
& \leqslant d+\left(\alpha_{i-1}-1\right)+\alpha_{i+1}-(k+1)=0 .
\end{aligned}
$$

Recalling that $d \geqslant 0$, this gives that $d=0$. This is the crucial consequence of the rows satisfying the strict triangle inequality.

Plugging this into (3) gives that $\alpha_{i-1}+\alpha_{i+1}-1 \leqslant k+1$. Thus, we have

$$
\alpha_{i-1}+\alpha_{i+1}-1 \leqslant k+1 \leqslant \alpha_{i} \leqslant \alpha_{i-1}+\alpha_{i+1}-1,
$$

meaning $\alpha_{i-1}+\alpha_{i+1}-1=k+1=\alpha_{i}$. This means that all $\alpha_{i}$ entries in the $(i+1)^{\text {st }}$ row of $A_{(i+1)}$ are $z$ 's, as desired.

In the following claim, we continue the proof of Theorem 21 by once again strengthening our assumptions regarding the entries of $A_{(i i+1)}$.

Claim 24. We can assume that the leftmost entry in the $(i-2)^{\text {nd }}$ row of $A_{(i i+1)}$ is an $r^{\prime}$, and that the rightmost entry in the $(i-1)^{\text {st }}$ row of $A_{(i+1)}$ is a $z$ (see Figure 12).

Proof of Claim 24. Let $s$ denote the leftmost entry in the $(i-2)^{n d}$ row of $A_{(i i+1)}$, and let $t$ denote the rightmost entry in the $(i-1)^{s t}$ row of $A_{(i i+1)}$ (see Figure 11). (Note that the $(i-1)^{s t}$ row cannot be the top row of $A_{(i i+1)}$, as this would require that $t=r^{\prime}=1$, which are incompatible with the assumptions that $d=0$ and that $A_{(i i+1)}$ is Yamanouchi.)

We now argue that we can assume $s=r^{\prime}$. First note that $t \neq r^{\prime}$, since if $t=r^{\prime}$, then the assumption that $d=0$ would imply that the prefix of the RRW ending immediately before $t$ contains one more $z$ than $r^{\prime}$, a violation of the Yamanouchi property. Therefore, $t>r^{\prime}$.

If $s<r^{\prime}$, then the $r^{\prime}$ in box $R^{\prime}$ can be swapped with $t$ to obtain an LR-tableau. If $s>r^{\prime}$, then we can perform the following two swaps to obtain an LR-tableau: swap the 


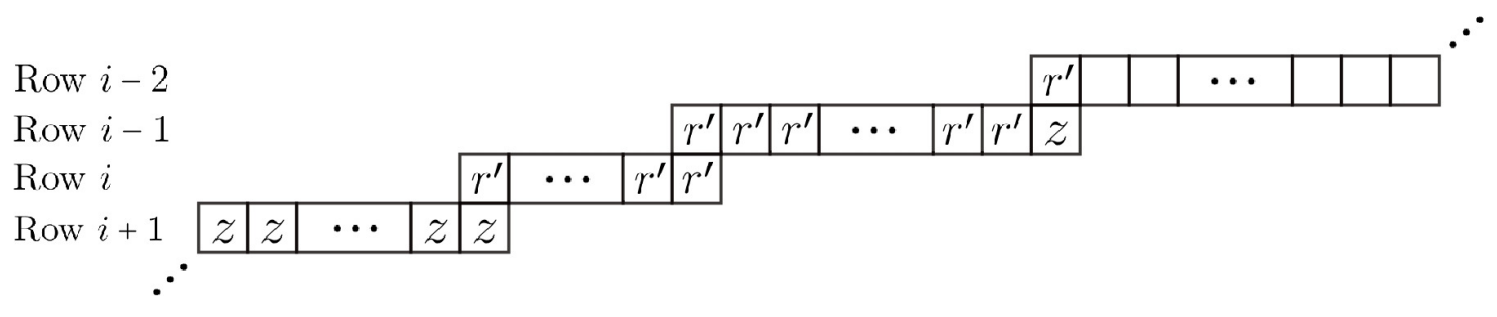

Figure 12: Assumed entries of $A_{(i+1)}$.

$r^{\prime}$ in box $R^{\prime}$ with $t$, and then swap the same $r^{\prime}$ with the $s$ above it. We can therefore assume that $s=r^{\prime}$.

Moreover, we argue that $t=z=r^{\prime}+1$. Recall that $d=0$; if $t \neq z$, then the prefix of the RRW of $A_{(i i+1)}$ formed by truncating immediately before $s$ is not Yamanouchi. These updated assumptions are shown in Figure 12.

We will now complete the proof of Theorem 21 by investigating $A_{(i i+1)}$, with content assumed to be as shown in Figure 12. In particular, we will scan $A_{(i i+1)}$ upwards from the $i^{\text {th }}$ row, arguing that we must eventually find an entry we can swap with the $r^{\prime}$ in either box $L^{\prime}$ or box $R^{\prime}$ of $A_{(i i+1)}$ to obtain an LR-tableau.

In what follows, a row of $A_{(i i+1)}$ will be called trivial if it is of length two and its entries are " $r$ ' $z$ " when reading from left to right across the row; otherwise, the row will be called nontrivial. There must be a nontrivial row among the first $i-2$ rows of $A_{(i i+1)}$, as otherwise the first letter of the RRW of $A_{(i i+1)}$ would be $z>r^{\prime} \geqslant 1$. Choose the maximal $j \leqslant i-2$ such that the $j^{\text {th }}$ row of $A_{(i i+1)}$ is nontrivial.

By our choice of $j$, all rows strictly between the $j^{\text {th }}$ and $(i-1)^{\text {st }}$ rows are trivial, so the rightmost box of the $(j+1)^{s t}$ row must contain a $z$ (even if $j=i-2$ ). Therefore, we have by the semistandardness of $A_{(i i+1)}$ that the leftmost entry $u$ of the $j^{\text {th }}$ row is at most $r^{\prime}=z-1$.

If $u<r^{\prime}$, then the $z$ in the rightmost box of the $(j+1)^{s t}$ row can be swapped with the $r^{\prime}$ in box $R^{\prime}$ of $A_{(i i+1)}$ to obtain a semistandard tableau $A_{(i i+1), z}$ (see Figure 13). Moreover, $A_{(i i+1), z}$ is Yamanouchi, since to obtain the RRW of $A_{(i i+1), z}$ from that of $A_{(i i+1)}$, one does not move $r^{\prime}$ in front of any $\left(r^{\prime}-1\right)$ 's, nor does one move $z$ behind any $(z+1)^{\prime} s$. We can therefore assume that $u=r^{\prime}$.

Let $v$ denote the entry to the right of $u$ in the $j^{\text {th }}$ row of $A_{(i i+1)}$ (see Figure 13). Recall that $d=0$ and that all rows strictly between the $j^{t h}$ and $(i-1)^{s t}$ rows are trivial. As a result, if $v=r^{\prime}$ as well, then the prefix of the RRW ending immediately before $v$ is not Yamanouchi. We can therefore assume that $v>r^{\prime}$.

Suppose that $\alpha_{j}>2$. Then $v$ is not the rightmost element of the $j^{\text {th }}$ row of $A_{(i i+1)}$, so we can swap it with the $r^{\prime}$ in box $R^{\prime}$ of $A_{(i i+1)}$ to obtain a semistandard tableau $A_{(i i+1), v}$. Moreover, $A_{(i i+1), v}$ is Yamanouchi, since to obtain its RRW from that of $A_{(i i+1)}$, one does not move $v$ behind any instances of $v+1$, nor does one move $r^{\prime}$ ahead of any instances of $r^{\prime}-1$. Therefore, let us assume that $\alpha_{j}=2$.

If the leftmost entry in the $(j-1)^{s t}$ row of $A_{(i i+1), v}$ (call it $\left.q\right)$ is less than $r^{\prime}$, then 


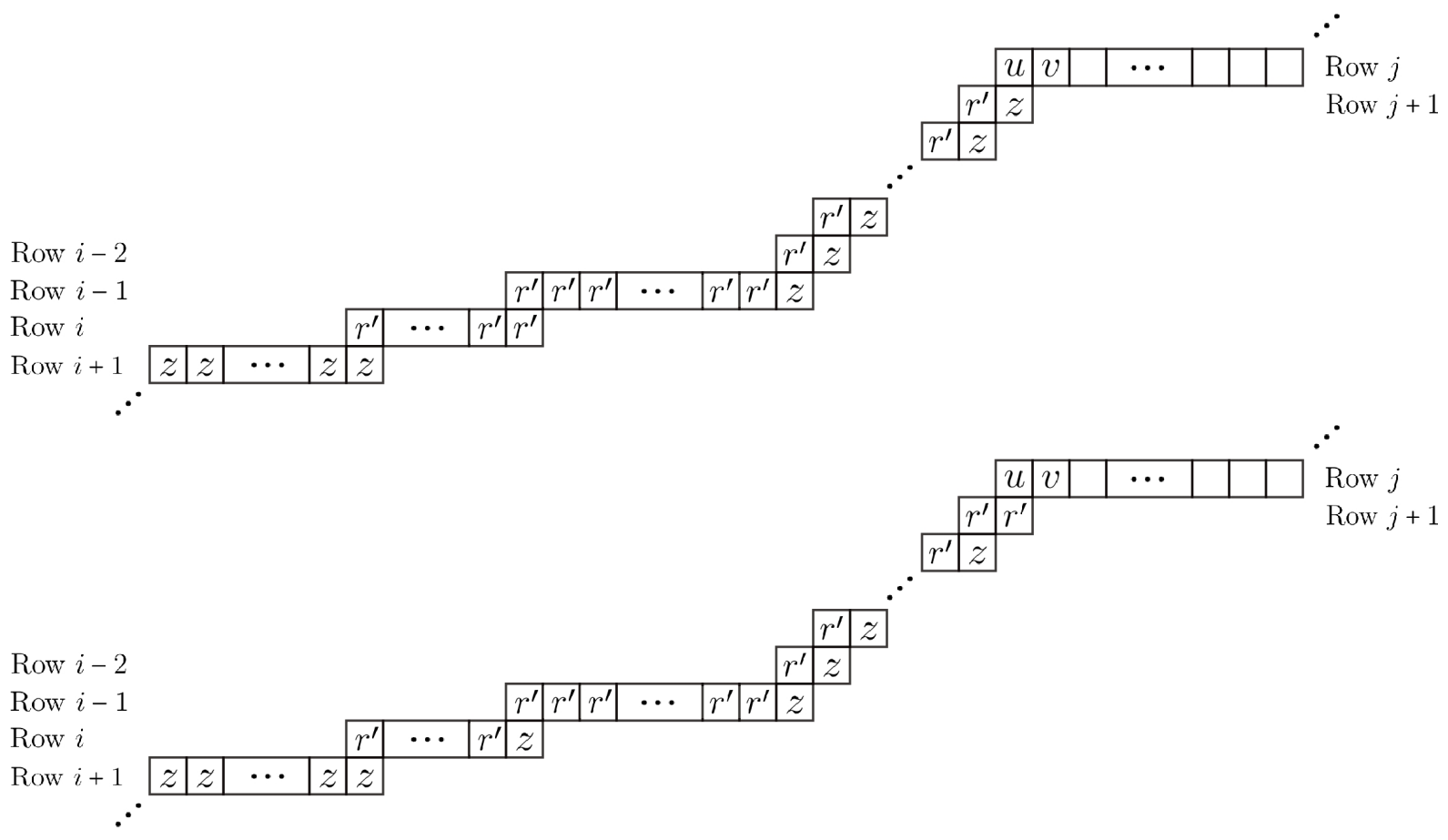

Figure 13: Assumed entries of $A_{(i+1)}$ (top) and $A_{(i i+1), z}$ (bottom).

$A_{(i i+1), v}$ is again an LR-tableau. Therefore, assume that $q \geqslant r^{\prime}$, in which case $A_{(i i+1), v}$ is not semistandard. If $q>r^{\prime}$, we can swap $q$ with the $r^{\prime}$ beneath it to obtain an LR-tableau. We can therefore assume that $q=r^{\prime}$.

We now return to considering the tableau $A_{(i i+1)}$, with the assumptions that $\alpha_{j}=2$ and $q=r^{\prime}$. Recall again that $d=0$ and that all rows strictly between the $j^{\text {th }}$ and $(i-1)^{\text {st }}$ rows of $A_{(i i+1)}$ are trivial. If $v \neq z$, then the prefix of the RRW of $A_{(i i+1)}$ ending immediately before $q$ is not Yamanouchi. Consequently, we have that $v=z$, contradicting our assumption that the $j^{\text {th }}$ row of $A_{(i i+1)}$ is nontrivial. Therefore we have already exhausted all possible cases, showing in each one how to obtain a ribbon LR-tableau of shape $\alpha_{(i i+1)}$ and content $\nu$. This completes the proof.

Since transpositions generate the symmetric group, Theorem 21 allows us to prove the following sufficient condition for a ribbon to have full equivalence class. This result generalizes the finding of McNamara and van Willigenburg that all equitable ribbons have full equivalence class [7, Theorem 1.5].

Theorem 25. Let $\alpha=\left(\alpha_{1}, \alpha_{2}, \ldots, \alpha_{m}\right)$ be a ribbon with each $\alpha_{i} \geqslant 2$ and $m \geqslant 3$. If all 3-subsets of $\left\{\alpha_{i}\right\}_{i=1}^{m}$ satisfy the strict triangle inequality, then $\alpha$ has full equivalence class.

Proof. Let $i \in\{1,2, \ldots, m-1\}$ be arbitrary. As noted above, it suffices to show that $[\alpha]=\left[\alpha_{(i i+1)}\right]$. If $\alpha_{i}=\alpha_{i+1}$, then $[\alpha]=\left[\alpha_{(i i+1)}\right]$ follows trivially. Thus, by Remark 2, we can assume without loss of generality that $\alpha_{i}>\alpha_{i+1}$. By assumption, $\alpha_{i}<\alpha_{i-1}+\alpha_{i+1}$, so Theorem 21 implies that $[\alpha] \subseteq\left[\alpha_{(i i+1)}\right]$. 
To show containment the other way, we consider the antipodal rotation

$$
\alpha^{\circ}=\left(\alpha_{1}^{\circ}, \alpha_{2}^{\circ}, \ldots, \alpha_{m}^{\circ}\right)=\left(\alpha_{m}, \alpha_{m-1}, \ldots, \alpha_{1}\right)
$$

of $\alpha$, with $\alpha_{0}^{\circ}=\infty$ for notational convenience. Set $j=m+1-i$, so that $\alpha_{j}^{\circ}=\alpha_{i}$ and $\alpha_{j-1}^{\circ}=\alpha_{i+1}$. Note that $2 \leqslant j \leqslant m$ since $1 \leqslant i \leqslant m-1$. Since $\left(\alpha_{(i, i+1)}\right)^{\circ}=\alpha_{(j-1 j)}^{\circ}$, we have by Remark 2 that $\left[\alpha_{(i i+1)}\right]=\left[\alpha_{(j-1 j)}^{\circ}\right]$. Hence, in light of Remark 2, it will suffice to show that $\left[\alpha_{(j-1 j)}^{\circ}\right] \subseteq\left[\alpha^{\circ}\right]$.

Since $\alpha_{i}>\alpha_{i+1}$, we have that $\alpha_{j}^{\circ}>\alpha_{j-1}^{\circ}$. Moreover, we have by assumption that $\alpha_{j}^{\circ}<\alpha_{j-1}^{\circ}+\alpha_{j-2}^{\circ}$. Thus, we have established that the ribbon $\alpha_{(j-1 j)}^{\circ}$ meets the conditions from Theorem 21 with respect to its $(j-2)^{n d},(j-1)^{s t}$, and $j^{\text {th }}$ rows. Since swapping $\alpha_{j}^{\circ}$ and $\alpha_{j-1}^{\circ}$ in $\alpha_{(j-1 j)}^{\circ}$ gives us back $\alpha^{\circ}$, Theorem 21 implies that $\left[\alpha_{(j-1 j)}^{\circ}\right] \subseteq\left[\alpha^{\circ}\right]$, as desired.

\section{A Necessary Condition}

In this section, we prove a necessary condition for a ribbon to have full equivalence class, which we conjecture to in fact be sufficient. Before we can state the condition, we need a lemma.

Lemma 26. Let $\alpha=\left(\alpha_{1}, \alpha_{2}, \ldots, \alpha_{m}\right)$ be a ribbon with $\alpha_{1} \geqslant \alpha_{2} \geqslant \ldots \geqslant \alpha_{m}$, each $\alpha_{i} \geqslant 2$ and $m \geqslant 3$. Then for any $1 \leqslant j \leqslant m$, any LR-tableau of shape $\alpha$ contains no more than $M_{j}:=\sum_{i=j}^{m} \alpha_{i}-(m-j)$ occurrences of $j$.

Proof. Fix a $j$ with $1 \leqslant j \leqslant m$. If $\alpha$ is an LR-tableau, then there cannot be any $j$ 's in the first $(j-1)$ rows of $\alpha$. In the remaining $m-j+1$ rows, there are $m-j$ pairwise-disjoint $2 \times 1$ rectangles (where consecutive rows overlap). At most one box in each of these rectangles can contain a $j$, by semistandardness. Therefore, at most $\sum_{i=j}^{m} \alpha_{i}-(m-j)=M_{j}$ boxes in $\alpha$ can contain a $j$, as desired.

Theorem 27. Let $\alpha=\left(\alpha_{1}, \alpha_{2}, \ldots, \alpha_{m}\right)$ be a ribbon with $\alpha_{1} \geqslant \alpha_{2} \geqslant \cdots \geqslant \alpha_{m}$, where each $\alpha_{i} \geqslant 2$, and $m \geqslant 3$. If $[\alpha]=\left[\alpha_{(j j+1)}\right]$, then $N_{j} \leqslant M_{j+1}$, where

$$
N_{j}:=\max \left\{k: \sum_{i \leqslant j: \alpha_{i}<k}\left(k-\alpha_{i}\right) \leqslant m-j-2\right\} .
$$

In particular, if $\alpha$ has full equivalence class, then, for all $1 \leqslant j \leqslant m-2$, we have $N_{j} \leqslant M_{j+1}$.

In fact, we conjecture that this condition is also sufficient:

Conjecture 28. Let $\alpha=\left(\alpha_{1}, \alpha_{2}, \ldots, \alpha_{m}\right)$ be a ribbon with $\alpha_{1} \geqslant \alpha_{2} \geqslant \cdots \geqslant \alpha_{m}$, where each $\alpha_{i} \geqslant 2$, and $m \geqslant 3$. Then $\alpha$ has full equivalence class if and only if $N_{j} \leqslant M_{j+1}$ for all $1 \leqslant j \leqslant m-2$.

Remark 29. Note that the condition from Conjecture 28, if it is indeed sufficient, would subsume the sufficient condition proved in Theorem 25. 
Remark 30. In general, to efficiently determine $N_{j}$, one can use the following algorithm:

1. Start with $k=\alpha_{j}$.

2. Set $s=0$. For each $\alpha_{i} \in\left\{\alpha_{1}, \alpha_{2}, \ldots, \alpha_{j}\right\}$ with $k>\alpha_{i}$, add $\left(k-\alpha_{i}\right)$ to $s$.

3. If $s>m-j-2$, then $N_{j}=k-1$. Otherwise, put $k=k+1$ and return to step 2 .

Remark 31. We always have $\alpha_{j} \leqslant N_{j} \leqslant \alpha_{j}+m-j-2$. In particular, $N_{j}=\alpha_{j}$ whenever $j=m-2$, while $N_{j}=\alpha_{j}+m-j-2$ if and only if $\alpha_{j} \leqslant \alpha_{j-1}-(m-j-2)$.

Remark 32. A much weaker but simpler version of our necessary condition is that $\alpha_{j}<$ $\sum_{i=j+1}^{m} \alpha_{i}$ for all $1 \leqslant j \leqslant m-2$, i.e. that each row is shorter than the sum of all the ones after it.

Our necessary condition of Theorem 27 is certainly less penetrable than the sufficient condition from Theorem 21, so before delving into the proof, we will illustrate the necessary condition with an example and a non-example. Additionally, the proof we will give is a constructive one, which we hope will make the above definition of $N_{j}$ more transparent.

Example 33. Let $\alpha=(10,8,6,5,4)$ be a ribbon. Then $\alpha$ does not satisfy our sufficient condition of Theorem 25 (as $10 \geqslant 5+4$ ). However, we now show that it does satisfy the necessary condition of Theorem 27. Since $m=5$ in this example, checking the necessary condition amounts to checking the inequalities corresponding to $j=1,2,3$ :

- We have

$$
N_{1}=\max \left\{k: \sum_{i \leqslant 1: \alpha_{i}<k}\left(k-\alpha_{i}\right) \leqslant 2\right\}=\max \{k:(k-10) \leqslant 2\}=12 .
$$

Since $12 \leqslant M_{2}=\sum_{i=2}^{5} \alpha_{i}-3=20$, the necessary inequality holds for $j=1$.

- We have

$$
N_{2}=\max \left\{k: \sum_{i \leqslant 2: \alpha_{i}<k}\left(k-\alpha_{i}\right) \leqslant 1\right\}=9
$$

since $9-8 \leqslant 1$, but $10-8>1$. Additionally, $9 \leqslant M_{3}=\sum_{i=3}^{5} \alpha_{i}-2=13$, so the inequality corresponding to $j=2$ holds.

- We have

$$
N_{3}=\max \left\{k: \sum_{i \leqslant 3: \alpha_{i}<k}\left(k-\alpha_{i}\right) \leqslant 0\right\}=6,
$$

since the summation when $k=6$ is the empty sum (and hence is zero), whereas $\sum_{i \leqslant 3: \alpha_{i}<7}\left(7-\alpha_{i}\right)=7-6=1>0$. Since $6 \leqslant M_{4}=\sum_{i=4}^{5} \alpha_{i}-0=9$, the inequality corresponding to $j=3$ holds. 
Non-Example 34. Let $\alpha=(13,10,5,4,3)$. Then

$$
N_{2}=\max \left\{k: \sum_{i \leqslant 2: \alpha_{i}<k}\left(k-\alpha_{i}\right) \leqslant 1\right\}=11 .
$$

Since $11>M_{3}=\sum_{i=3}^{5} \alpha_{i}-2=10$, this shows that the necessary inequality does not hold for $j=2$. As a result, Theorem 27 tells us that $\alpha$ does not have full equivalence class.

Having gained some familiarity with the necessary condition, we now turn towards its proof, which we begin with a lemma.

Lemma 35. Let $\alpha=\left(\alpha_{1}, \alpha_{2}, \ldots, \alpha_{m}\right)$ be a ribbon with $\alpha_{1} \geqslant \alpha_{2} \geqslant \cdots \geqslant \alpha_{m}$, where each $\alpha_{i} \geqslant 2$, and $m \geqslant 3$. If $N_{j}>M_{j+1}$ for some $j \in\{1,2, \ldots, m-2\}$, then $\alpha_{j}>\alpha_{j+1}$.

Proof. The inequality $N_{j} \geqslant M_{j+1}+1$ implies that

$$
\alpha_{j+1} \leqslant N_{j}+(m-j-2)-\sum_{i=j+2}^{m} \alpha_{i} \leqslant \alpha_{j}+2(m-j-2)-\sum_{i=j+2}^{m} \alpha_{i}<\alpha_{j},
$$

where the second inequality comes from the upper bound on $N_{j}$ given in Remark 31 , and the third inequality follows from the assumption that all rows are at least two boxes long.

Proof of Theorem 27. We prove the contrapositive. Fix $j \in\{1,2, \ldots, m-2\}$ such that $N_{j}>M_{j+1}$. We will show that there is no ribbon LR-tableau of shape $\alpha$ and content $\nu$ satisfying $\nu_{j+1}=M_{j+1}+1$; we will then exhibit a ribbon LR-tableau of shape $\alpha_{(j j+1)}$ with such content.

From Lemma 26, we have that the maximum number of $(j+1)$ 's that can occur in an LR-tableau of shape $\alpha$ is at most $M_{j+1}$. Therefore, there is no LR-tableau of shape $\alpha$ and content $\nu$ satisfying $\nu_{j+1}=M_{j+1}+1$. However, the following argument shows that there does exist a ribbon LR-tableau with such content.

Fill $\alpha_{(j j+1)}$ as follows (we'll call the resulting tableau $A$ ). Fill the $i^{\text {th }}$ row entirely with $i$ 's for $i \leqslant j$. Put $(j+1)$ 's in the $\alpha_{j+1}$ rightmost boxes of the $(j+1)^{s t}$ row and fill the remaining boxes in this row with $j$ 's. By Lemma 35 we have that $\alpha_{j}>\alpha_{j+1}$, meaning the leftmost entry of the $(j+1)^{\text {st }}$ row in this filling is a $j$.

We now fill the remaining $m-j-1$ rows with as many $(j+1)$ 's as possible (while maintaining semistandardness, but perhaps not the Yamanouchi property, although we will show with the upcoming arguments that it indeed is Yamanouchi); put $(j+1)$ 's in all but the leftmost box of the next $m-j-2$ rows, as well as in every box in the last row. Now the only empty boxes are the leftmost boxes in rows $j+2, j+3 \ldots, m-2, m-1$ (see Figure 14). We will call these remaining boxes critical boxes.

Fill the critical boxes from top to bottom according to the following algorithm: in each box, put the largest integer $\leqslant j$ such that the prefix of RRW through that box remains Yamanouchi. In practice, this means we will use exclusively $j$ 's until the number of $j$ 's in 


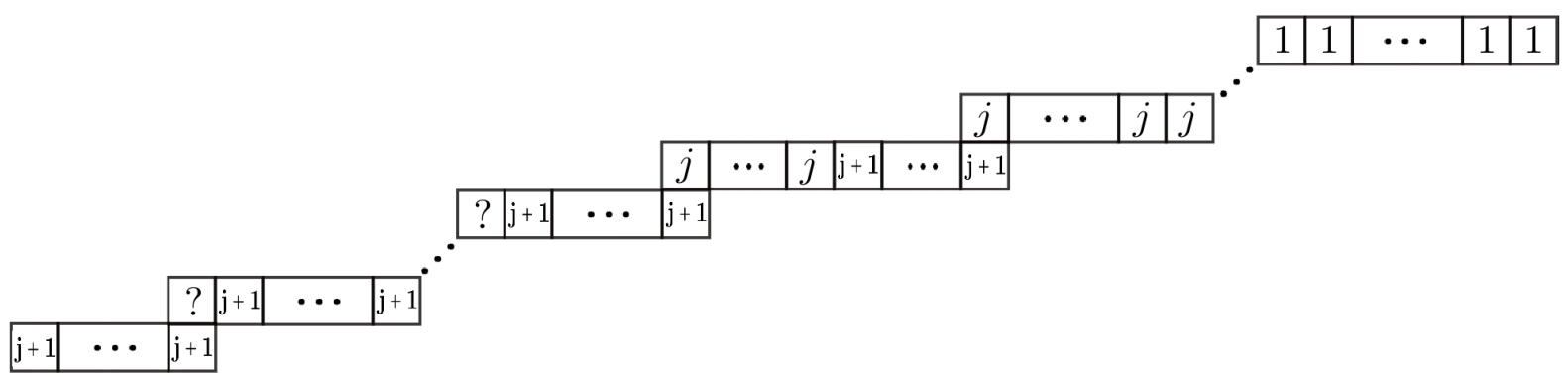

Figure 14: The filling of $A$, with critical boxes indicated by "?".

the tableau equals the number of $(j-1)$ 's. Then, we will alternate between $(j-1)$ 's and $j$ 's until the both the number of $j$ 's and of $(j-1)$ 's equals the number of $(j-2)$ 's. At this point, we rotate between placing $j$ 's, $(j-1)$ 's, and $(j-2)$ 's until the number of each of these equals the number of $(j-3)$ 's. We continue in this manner until all boxes have been filled. Towards proving that the resulting tableau $A$ is Yamanouchi, we first show that it contains exactly $N_{j} j$ 's.

First we define a round. Let the variables $c_{1}, c_{2}, \ldots, c_{m-j-2}$ represent the entries in the critical boxes, from top to bottom. Let $J=\left\{s: c_{s}=j\right\}$. Now partition $\{1,2, \ldots, m-$ $j-2\}$ into rounds, where each round $s_{1}, s_{1}+1, \ldots, s_{1}+d$ is a consecutive subsequence of $1,2, \ldots, m-j-2$ such that $s_{1}+d \in J$ and $p \notin J$ for all $s_{1} \leqslant p \leqslant s_{1}+d-1$ (i.e. a round ends if and only if a $j$ is encountered, counting from 1 up to $m-j-2$ ).

Claim 36. If r rounds can be completed before reaching the bottom, then at the end of the $r^{\text {th }}$ round, we have filled exactly $\sum_{i \leqslant j: \alpha_{i}<\alpha_{j}+r}\left(\alpha_{j}+r-\alpha_{i}\right)$ critical boxes. In particular, after the $r^{\text {th }}$ round, each number $i \leqslant j$ such that $\alpha_{i} \leqslant \alpha_{j}+r$ has occurred in exactly $\alpha_{j}+r-\alpha_{i}$ critical boxes.

Proof of Claim 36. We will use induction on $r$. The claim trivially holds when $r=0$. Now consider an arbitrary $r>0$ (such that $r$ rounds can be completed before reaching the bottom) and assume the claim holds for $r-1$. In the $r^{\text {th }}$ round, we will write every number that was used in the $(r-1)^{s t}$ round one additional time, as well as any number $\ell$ satisfying $\alpha_{\ell}=\alpha_{j}+r-1$. Therefore, the latter numbers $\ell$ will each fill exactly one critical box after $r$ rounds, as is appropriate since, by choice of $\ell, \alpha_{j}+r-\alpha_{\ell}=1$. All numbers which appeared in the $(r-1)^{s t}$ round have now occurred in a critical box one more time than before. For a fixed number $i$, by the induction hypothesis, this is $\alpha_{j}+(r-1)-\alpha_{i}+1=\alpha_{j}+r-\alpha_{i}$ times. This completes the proof of Claim 36.

Clearly the number of $j$ 's in $A$ is $\alpha_{j}$ plus the number of rounds executed before running 
out of critical boxes. That is, if $\nu_{j}$ is the number of $j$ 's in $A$, then

$$
\begin{aligned}
\nu_{j} & =\alpha_{j}+\max \left\{r: \sum_{i \leqslant j: \alpha_{i}<\alpha_{j}+r} \alpha_{j}+r-\alpha_{i} \leqslant m-j-2\right\} \\
& =\max \left\{k: \sum_{i \leqslant j: \alpha_{i}<k}\left(k-\alpha_{i}\right) \leqslant m-j-2\right\}=N_{j} .
\end{aligned}
$$

In particular, $\nu_{j}=N_{j}$.

By construction, the number of $(i+1)$ 's never surpasses the number of $i$ 's in the RRW of $A$, since $1 \leqslant i \leqslant j-1$. Semistandardness is also clear by construction, so all that is left to check is that the number of $(j+1)$ 's never overtakes the number of $j$ 's in the RRW of A.

It is clear that the number of $(j+1)$ 's does not surpass the number of $j$ 's in the first $j+1$ rows of the tableau. Since each of the remaining rows has at least as many $(j+1)$ 's as $j$ 's and the last row consists entirely of $(j+1)$ 's, the number of $(j+1)$ 's can only overtake the number of $j$ 's if the total number of $(j+1)$ 's in $A$ is greater than the total number of $j$ 's in $A$ (that is, if $\nu_{j+1}>\nu_{j}$ ). Therefore, it suffices to show that $\nu_{j}=N_{j} \geqslant \nu_{j+1}$. Indeed, observe that $\nu_{j+1}=M_{j+1}+1$, meaning this inequality follows immediately from our assumption that $N_{j} \geqslant M_{j+1}+1$. This completes the argument that $A$ (which has shape $\left.\alpha_{(j j+1)}\right)$ is an LR-tableau.

Corollary 10 now gives that $\nu$ is in the support of $\alpha_{(j j+1)}$, but is not in the support of $\alpha$, completing the proof.

We have proven that the condition in Theorem 27 is necessary for a ribbon to have full equivalence class. In fact, in [3], the authors (along with Tran) proved that this condition is both necessary and sufficient for ribbons with three or four rows to have full equivalence class. (When $m=3$, our necessary and sufficient conditions coincide, while the $m=4$ case requires additional analysis/case-work.) In addition, we have verified by computer that this condition is sufficient for $m=5, m=6$, and $m=7$ for certain $n$ (where $n$ is the number of boxes in the diagram).

\section{Concluding Remarks and Future Work}

In this paper, we have presented substantial progress towards classifying when a permutation $\pi \in S_{m}$ of row lengths of a ribbon $\alpha$ produces a ribbon $\alpha_{\pi}$ with the same Schur support as $\alpha$. However, there are several ways in which we would like to generalize our results so as to obtain a more complete answer to the following central question:

Problem 37. Given two skew shapes $\lambda_{1} / \mu_{1}$ and $\lambda_{2} / \mu_{2}$, when is it the case that $\left[\lambda_{1} / \mu_{1}\right]=$ $\left[\lambda_{2} / \mu_{2}\right]$ ?

Although Problem 37 has proven to be difficult in general, one potentially feasible step forward would be to develop analogues of our main results for skew shapes other than ribbons. 
To fully understand when ribbons have equal Schur supports, we would first like to prove Conjecture 28, which would completely classify when a ribbon has full equivalence class. We would then like to investigate support equalities among ribbons which do not have full equivalence class. Namely, we pose the following open question:

Problem 38. Given a ribbon $\alpha$, for which $\pi \in S_{m}$ do we have $[\alpha]=\left[\alpha_{\pi}\right]$ ?

Theorem 21 offers progress towards answering this question, giving a sufficient condition for the support containment $[\alpha] \subseteq\left[\alpha_{(i i+1)}\right]$ for any $1 \leqslant i \leqslant(m-1)$.

Our final two questions take a step back from support equality to wonder how our work might extend, or relate, to the domains of support containment and comparison under the Schur-positivity order.

Problem 39. When a ribbon fails to have full equivalence class, are its permutations still comparable to each other under the support containment order? If so, is there a criterion which prescribes the direction of the containment for each pair of the ribbon's permutations?

Problem 40. Our results show that ribbons with full equivalence class are, in a sense, "more equitable" than ribbons without full equivalence class (meaning the former's row lengths are more balanced than the latter's). Since equitable ribbons are maximal with respect to Schur-positivity order (as found by McNamara and van Willigenburg in [7]), we ask: does full equivalence class predict a higher place in the Schur-positivity order? That is, if $\alpha$ and $\beta$ are ribbons with the same number of rows, columns, and boxes, such that $\alpha$ has full equivalence class, but $\beta$ does not, does it follow that $s_{\alpha}-s_{\beta}$ is Schur-positive? If this is too much to ask, then do we at least have that $[\beta] \subseteq[\alpha]$ ?

\section{Acknowledgements}

This research was performed as part of the 2017 University of Minnesota, Twin Cities Combinatorics REU, and was supported by NSF RTG grant DMS-1148634 and by NSF grant DMS-1351590. We would like to thank Victor Reiner, Pavlo Pylyavskyy, and Galen Dorpalen-Barry for their advice, mentorship, and support. We are also grateful to Sunita Chepuri for her help in finding a reference on $R$-matrices.

\section{References}

[1] O. Azenhas, R. Mamede. A classification of monotone ribbons with full Schur support with application to the classification of full equivalence class, ArXiv e-prints (Dec. 2018). arXiv:1812.04705v1.

[2] L. J. Billera, H. Thomas, and S. van Willigenburg. Decomposable compositions, symmetric quasisymmetric functions and equality of ribbon Schur functions, Adv. Math. 204 (2006), 204-240. 
[3] M. Gaetz, W. Hardt, S. Sridhar, and A. Tran. Support equalities of ribbon schur functions, http://www-users.math.umn.edu/ reiner/REU/GaetzHardtSridharTran_ prob1_2017.pdf.

[4] R. Inoue, A. Kuniba, and T. Takagi. Integrable structure of box-ball systems: crystal, Bethe ansatz, ultradiscretization and tropical geometry, J. Phys. A 45 (2012) 7.

[5] D.E. Littlewood and A.R. Richardson. Group characters and algebra, Philos. Trans. R. Soc. Lond. Ser. A Math. Phys. Eng. Sci. 233 (1934) 721-730, 99-141.

[6] P. R. W. McNamara. Necessary conditions for Schur-positivity, J. Algebraic Combin. 28 (2008) 4, 495-507.

[7] P. R. W. McNamara and S. van Willigenburg. Maximal supports and Schur-positivity among connected skew shapes, European J. Combin. 33 (2012) 6, 1190-1206.

[8] P. R. W. McNamara and S. van Willigenburg. Towards a combinatorial classification of skew Schur functions, Trans. Amer. Math. Soc. 361 (2009) 8, 4437-4470.

[9] A. Nakayashiki, and Y. Yamada. Kostka polynomials and energy functions in solvable lattic models, Selecta Math. (N.S.) 3 (1997), 547-599.

[10] V. Reiner, K. M. Shaw, and S. van Willigenburg. Coincidences among skew Schur functions, Adv. Math. 216 (2007) 1, 118-152.

[11] R. Stanley. Enumerative Combinatorics. Vol. 2, volume 62 of Cambridge Studies in Advanced Mathematics. Cambridge University Press, Cambridge, 1999. 